(2) Open Access Full Text Article

REVIEW

\title{
Some aspects of allogeneic stem cell transplantation in patients with myelodysplastic syndrome: advances and controversy
}

This article was published in the following Dove Press journal:

Stem Cells and Cloning: Advances and Applications

4 December 2014

Number of times this article has been viewed

\section{Olga Blau \\ Igor Wolfgang Blau}

Department of Hematology, Oncology and Tumor Immunology, Charité - Universitätsmedizin Berlin, Berlin, Germany
Correspondence: Olga Blau Charité Universitätsmedizin Berlin, 30 Hindenburgdamm, Berlin 12200, Germany Tel +4930405026492 $\mathrm{Fax}+4930405026618$ Email olga.blau@charite.de
Abstract: Myelodysplastic syndrome (MDS) is a heterogeneous group of myeloid disorders. MDS remains a disease of elderly patients; moreover, the incidence of high risk MDS is proportionally greater in elderly patients, with increased frequency of secondary acute myeloid leukemia, as well as adverse cytogenetic abnormalities. Allogeneic stem cell transplantation is a therapeutic approach with known curative potential for patients with MDS that allows the achievement of long-term disease control. Numerous controversies still exist regarding transplantation in MDS: timing of transplantation, disease status at transplantation and comorbidity, conditioning intensity, pretransplant therapy, and stem cell source. Various transplant modalities of different intensities and alternative donor sources are now in use. Current advances in transplant technology are allowing the consideration of older patients. This should result in a greater number of older patients benefiting from this potentially curative treatment modality. Despite advances in transplantation technology, there is still considerable morbidity and mortality associated with this approach. Nevertheless, with the introduction of reduced-intensity conditioning and thereby reduced early mortality, transplant numbers in MDS patients have significantly increased. Moreover, recent new developments with innovative drugs, including hypomethylating agents, have extended the therapeutic alternatives for MDS patients. Hypomethylating agents allow the delay of allogeneic stem cell transplantation by serving as an effective and well-tolerated means to reduce disease burden.

Keywords: myelodysplastic syndrome, allogeneic stem cell transplantation, reduced-intensity conditioning

\section{Introduction}

Myelodysplastic syndrome (MDS) is a heterogeneous group of myeloid disorders characterized by peripheral blood cytopenias and increased risk of transformation to acute myelogenous leukemia (AML). ${ }^{1}$ Overall survival (OS) after diagnosis of MDS varies from a few months to several years. Among patients under the age of 60 years, median OS is 4.6 years; it is significantly lower for those diagnosed after the age of 60 years. ${ }^{1,2}$ Allogeneic stem cell transplantation (alloSCT) is a therapeutic approach with known curative potential for patients with MDS that allows the achievement of long-term disease control.

However, various controversies still exist regarding transplantation in MDS: timing of transplantation, disease status at transplantation and comorbidity, conditioning intensity, pretransplant therapy, and stem cell source. Modern transplant modalities of different intensities and alternative donor sources are now in use. Current advances in transplant technology are allowing the consideration of older patients..$^{3,4}$ This should 
result in a greater number of older patients benefiting from this potentially curative treatment modality.

Despite advances in transplantation technology, there is still considerable morbidity and mortality associated with this approach. ${ }^{5}$ Nevertheless, with the introduction of reduced-intensity conditioning (RIC) and thereby a reduction in early mortality, transplant numbers in MDS patients have significantly increased. ${ }^{6,7}$

Recent new developments with innovative drugs, including hypomethylating agents (HMAs), have extended the therapeutic alternatives for MDS patients. HMAs offer a "bridge" to or allow delay of alloSCT by serving as an effective and well-tolerated means to reduce disease burden. ${ }^{8,9}$

Determining the optimal time of alloSCT for MDS patients has proven difficult. Early studies suggested that patients with MDS had the best outcomes if they were transplanted early in the course of their disease..$^{10,11}$ The outcomes of alloSCT for MDS patients are essentially dependent on disease-related parameters, such as cytopenia, karyotype, percentage of blast cells in the bone marrow, and patientrelated factors reflecting comorbidities. The selection of an appropriate transplant candidate has undergone modifications over the last decade. This was facilitated by improving the understanding of the risk of disease, using modified modern therapy approaches that advance survival, and the more opportunities for alloSCT in the elderly with the advent of RIC and nonmyeloablative techniques.

\section{Prognostic scoring system, IPSS and WPSS}

Significant assistance in identifying patients who will benefit from alloSCT was provided by the International Prognostic Scoring System (IPSS), which is based on clinical, morphologic, and cytogenetic characteristics. ${ }^{2}$ Greenberg et al showed that the major variables having an impact on disease outcome for evolution to AML were cytogenetic abnormalities, percentage of bone marrow blasts, and number of cytopenias; for survival, in addition to the aforementioned factors, were additionally included age and sex. ${ }^{2}$

The IPSS defines the three cytogenetic risk categories: favorable (normal karyotype, isolated del(5q), del(20q), and loss of the $\mathrm{Y}$ chromosome), unfavorable (any abnormality of chromosome 7 and complex aberrations), and intermediate (any other abnormality, neither favorable nor unfavorable). ${ }^{2}$ During the last 15 years, increasing knowledge and higher numbers of cases have helped to improve the quality of cytogenetic prognosis. ${ }^{12}$ Recently, the IPSS has undergone revisions (IPSS-R) that take into consideration different subgroups of cytogenetic abnormalities and the depth of cytopenias, which led to the expansion of risk categories into five groups. ${ }^{13}$ The IPSS-R classifies cytogenetic information in MDS into five categories, "very good", "good", "intermediate", "poor", and "very poor" risk groups. ${ }^{14}$ The cytogenetic risk groups correspond to median OS of $61,49,26,16$, and 6 months, respectively. ${ }^{14}$ The probability of relapse increases progressively with increasing of the IPSS or WPSS (the World Health Organization [WHO] classification-based PSS) scores. ${ }^{15}$ The presence of poor-risk cytogenetic abnormalities has been associated with a significantly poorer outcome posttransplantation. Recently, a monosomal karyotype was shown to be a more accurate predictor of poor outcome in AML. ${ }^{16}$ The negative predictive power of the monosomal karyotype was shown to be slightly higher than that of a complex karyotype for progression-free survival after alloSCT. ${ }^{17}$

The IPSS-R demonstrated the depth of cytopenia as a useful prognostic factor. ${ }^{13}$ Previously, it was shown that the degree of anemia is an important factor of poor clinical outcomes in MDS..$^{18,19}$ Underlying this finding, chronic anemia may contribute to the high nonleukemic mortality related to cardiac disease in MDS patients. ${ }^{19}$

Although alloSCT could be considered in MDS patients, careful patient selection based on a thoughtful assessment of risk and benefit factors is important. ${ }^{20-22} \mathrm{~A}$ Markov decision analysis published in 2004 found that better OS achieved patients with IPSS intermediate 2 and high risk if they proceed to alloSCT immediately, while patients with low and intermediate 1 IPSS scores achieved better life expectancy by delaying alloSCT to the time of leukemic progression. ${ }^{11}$ This analysis included patients younger than 60 years who had undergone myeloablative conditioning (MAC) before using HMA therapy. To address these limitations, another Markov decision analysis with quality-of-life investigation was recently published based on evaluation of MDS patients aged $60-70$ years. ${ }^{23}$ The authors concluded that for de novo MDS patients with IPSS low- or intermediate 1-risk groups, nontransplantation approaches were preferred. In contrast, for those patients in the intermediate 2- and high IPSS-risk categories, RIC alloSCT offered an advantage in terms of OS and quality-adjusted survival. Patients in the intermediate 1-risk group only had a slight gain in life expectancy if alloSCT was delayed, and therefore decisions should probably be made on an individual basis in these patients, depending on platelet or neutrophil counts. ${ }^{11,24}$ A study published in 2008 retrospectively evaluated the impact of the WHO classification and WPSS on the outcome of patients who had undergone allogeneic hematopoietic 
SCT. ${ }^{15}$ The data suggest that for alloSCT lower-risk patients (WPSS risk score), 5-year OS was demonstrated for $80 \%$ of them. With increasing WPSS scores, the probability of 5 -year survival after alloSCT declined progressively to $65 \%$ (intermediate risk), 40\% (high risk), and 15\% (very high risk). ${ }^{15}$ Furthermore, recent data suggests that younger patients with low risk often have an extended period of time before significant disease progression. ${ }^{11,20,25}$ An early alloSCT in lower-risk MDS (low, intermediate 1) actually leads to life-years lost. The authors recommended upfront transplantation for patients with intermediate 2- or high-risk disease and delayed transplant for low-risk and intermediate 1 patients until disease progression but prior to leukemic transformation. ${ }^{11,26}$ Most experts and practice guidelines generally recommend earlier alloSCT for patients with intermediate 2- or high-risk IPSS scores. ${ }^{24,27}$

The Global MD Anderson Scoring System was proposed as a model, incorporating such patient-related factors as age and performance status. It accounts for anemia, transfusion burden, karyotype, and probably most importantly severity of thrombocytopenia and leukopenia. ${ }^{28}$

Additional, IPSS intermediate 1 patients harboring poorrisk cytogenetics, severe thrombocytopenia, or red blood cell-transfusion dependency might also be considered for hematopoietic alloSCT on an individual basis. ${ }^{4}$

\section{Age of patients}

Age is an important factor determining the decision about alloSCT. Although there is a clear agreement on the recommendation of alloSCT for higher-risk younger MDS patients, there is considerable controversy regarding elderly patients. Approximately $75 \%$ of patients with MDS are older than 60 years. The definition of "old age" in alloSCT varies in different studies, most often referring to patients aged 50-70 years. $^{29}$

Essentially, the incidence of poor-risk MDS is proportionally greater in elderly patients, with increased frequency of secondary AML, as well as adverse cytogenetic abnormalities. ${ }^{30,31}$ Early registry studies have demonstrated a significant correlation between advanced age and poor outcome. ${ }^{30-34}$ Kuendgen et al presented data on 232 patients less than 50 years of age and 2,496 patients more than 50 years of age, and showed that $42 \%$ of the patients younger than 50 years and only $8 \%$ of the older patients had undergone alloSCT. However, improvements in supportive care and importantly the use of RIC for alloSCT have substantially improved the outcome of transplantation among older patients, and currently there is no clear upper age limit. ${ }^{25}$
Later, Lim et al published a comparison of outcomes of 1,333 MDS patients older than 50 years. ${ }^{35}$ In this analysis, the disease stage at the time of transplantation, but not recipient age or the intensity of the conditioning regimens, was the most important factor influencing outcomes. ${ }^{35}$

In accordance with European LeukemiaNet recommendations (2013), fit patients up to age 65-70 years with IPSS intermediate 2 or high risk and those with IPSS intermediate 1 risk with excess blasts or poor-risk cytogenetics are candidates for alloSCT. ${ }^{36}$

\section{Comorbidity as prognostic variation}

The impact of age is a major prognostic parameter for OS. ${ }^{13}$ This effect has previously been shown with the IPSS and in other studies. ${ }^{2,13,37}$ In addition, aging is associated with an increasingly high risk of developing comorbidity, and a high prevalence of comorbid diseases has indeed been reported in MDS patients. ${ }^{38,39}$

Patient-related factors like chronic comorbid conditions, poor performance status, and abnormal organ function may also influence the outcome, especially survival in MDS patients. ${ }^{40}$ The Hematopoietic Cell Transplantation Comorbidity Index was established to estimate outcomes of patients specifically diagnosed with MDS or AML and given hematopoietic SCT. ${ }^{41}$

Various studies have shown that in elderly patients with a mean age of 70 years, comorbidity is also an important additional prognostic factor for survival. ${ }^{40-42}$ Recently, Sperr et al published an analysis of 519 de novo MDS patients with a median age of 71 years. The data indicated that comorbidity should be considered an important coverable in risk assessment in MDS. However, the progression of the disease depends on the biologic properties of the clone, rather than on the comorbidity status of the patient. ${ }^{40}$

In 2010, the MDS-specific Comorbidity Index (MDS-CI) was developed by the Italian MDS study group. ${ }^{39}$ Later, Zipperer et al presented a retrospective study of 1,161 patients, and suggested the use of the MDS-CI to assess concomitant diseases, since it shows high stability in the hands of different research groups. ${ }^{42}$

\section{Additional risk factors}

In addition, certain supplementary prognostic factors should be considered for alloSCT, such as refractory life-threatening cytopenias, ${ }^{1,43,44}$ the degree of transfusion dependency, and the related problem of transfusional iron overload. ${ }^{45}$ Some other disease-related variables, such as $\beta_{2}$ microglobulin, lactate dehydrogenase, bone marrow fibrosis, and the 
presence of small numbers of circulating blast cells, might be useful for improving the accuracy of predicting early disease progression. ${ }^{24}$

Although bone marrow fibrosis is not included in current prognostic risk scales, such as the IPSS and the WPSS, ${ }^{46}$ some investigators recommend more aggressive treatment for patients with bone marrow fibrosis. ${ }^{47}$ Kröger et al analyzed the impact of bone marrow fibrosis on outcome after alloSCT in MDS patients reported to the European Group for Blood and Marrow Transplantation registry. ${ }^{48}$ The authors concluded that only severe bone marrow fibrosis affects survival because of a higher risk of relapse, while MDS patients with mild or moderate bone marrow fibrosis have an outcome comparable to that of MDS patients without bone marrow fibrosis. ${ }^{48}$

Several groups have identified iron overload, in terms of raised pretransplant serum ferritin levels, as an independent adverse prognostic factor for patients undergoing MAC alloSCT. ${ }^{49-51}$ It has been reported that iron overload increases the risk of veno-occlusive disease, hepatic dysfunction, and infections after transplantation. ${ }^{52}$ It has also been shown that patients with elevated ferritin levels in the pretransplant period had poor survival, due especially to infections in alloSCT. ${ }^{53}$ The hyperferritinemia may be treated with oral deferasirox in the posttransplant, which can be an effective method of iron chelation. ${ }^{54}$

\section{Gene mutations}

Recently discovered important prognostic gene mutations (EZH2, SRSF2, $A S X L 1$ ), involving such epigenetic regulators as TET-2, IDH, and DNMT3A, and gene-expression aberrations (TP53) can define a subgroup of MDS patients with IPSS low or intermediate 1 who actually have poor outcomes. ${ }^{54-58}$ Bejar et al confirmed that mutations in the $E Z H 2$ gene are associated with disease subtypes, clinical features, and significantly shorter OS. ${ }^{55}$ Additionally, Thol et al showed a negative prognostic impact of SRSF2 mutations in MDS. ${ }^{56}$ Different mutations may become useful for clinical risk stratification and treatment decisions in the future. Determining an accurate prognosis is critical for the care and treatment of patients with MDS.

\section{Conditioning}

The impact of conditioning intensity on disease control remains controversial. Some authors report that MAC offers improved disease control, ${ }^{59}$ but often at the expense of increased treatmentrelated mortality (TRM). ${ }^{60-63}$ Two MAC regimens combining either cyclophosphamide and fractionated total body irradiation or busulfan and cyclophosphamide are used most widely.
In MAC transplantation, IPSS risk is correlated with MDS relapse and disease-free survival. ${ }^{64} \mathrm{TRM}$ is $35 \%-80 \%$, varying with age and other factors. ${ }^{6,10}$ Cutler et al documented that for patients 18-60 years of age with intermediate 2/high IPSS MDS, early MAC transplantation provides maximal qualityadjusted survival. ${ }^{11}$ Warlick et al reported that patients receiving MAC had a lower risk of relapse, particularly those in complete remission or with $<5 \%$ blasts. ${ }^{60}$ This finding contrasts with the data published by Scott et al, in which the authors found no difference in relapse rates in patients with complete remission and less than $5 \%$ of blasts prior to alloSCT between those who received either MAC or non-MAC. ${ }^{6}$

AlloSCT is associated with excessive procedure-related toxicity. ${ }^{35,65}$ Considerable risk of TRM and disease relapse limit long-term OS. ${ }^{66-68}$ Results from selected studies report prolonged disease-free survival in about $30 \%-50 \%$ of patients. ${ }^{69}$ The risk of organ toxicities has limited the use of high-dose regimens to younger patients in good medical condition. To avoid this limitation, a non-MAC regimen and widely used RIC regimens for alloSCT were developed. The regimen relies on graft-versus-leukemia (GVL) effects to cure cancer. Non-MAC regimens are usually based on 2 Gy of total body irradiation. RIC regimens contain treosulfan, busulfan, fludarabine, or melphalan. Various RIC regimens have been developed by several investigators in the last 15 years. ${ }^{70-74}$ This regimen has allowed the extension of alloSCT to a previously unserved population of older patients or those with clinically significant comorbidities. Since the introduction of RIC regimens has led to a significant reduction in TRM, the relapse has become the leading impediment to the achievement of long-term survival in transplanted MDS patients. ${ }^{5,43,71}$ The principal aim of RIC is to minimize toxicity associated with MAC regimens and to harness the GVL effect. RIC regimens depend largely upon intensive immune suppression either during conditioning and/ or after stem cell infusion to facilitate donor engraftment and establish complete donor chimerism. ${ }^{75}$

It is known that $75 \%$ of patients with MDS are older than 60 years at diagnosis, and are typically not considered MAC transplantation candidates. ${ }^{68,76}$ In patients older than 60 years, RIC transplantation is potentially curative, but is also associated with mortality risk. ${ }^{68}$ Different groups of authors reported that TRM was 26\%-41\%, with long-term MDS/AML survival of $27 \%-54 \% .^{6,61,77}$ RIC transplantation in older patients remains uncertain, because MDS prognosis differs from that of younger patients, and RIC and MAC transplantation risks and benefits may also differ. ${ }^{68}$

Lim et al demonstrated that an RIC regimen using fludarabine, busulfan, and alemtuzumab enabled high engraftment 
rates, with a low incidence of graft-versus-host disease (GVHD) and durable long-term survival. ${ }^{76}$ Advanced age does not appear to be a contraindication to RIC alloSCT, even with the use of unrelated donors, and identification of pretransplant comorbidities may help to better identify patient eligibility for transplantation.

\section{Donor sources}

The donor sources tested with regard to peripheral blood stem cells, bone marrow, or umbilical cord blood yielded similar outcomes. The data from Lim et al suggest that in the absence of a suitable HLA-matched related donor, RIC volunteer unrelated donor alloSCT offers a comparable alternative for MDS patients with progressive disease. ${ }^{76}$ These data and others suggest that unrelated donor transplantations offer the possibility of long-term survival in selected patients with high-risk MDS..$^{9,76}$

The results demonstrate strong negative effects of mismatching for either HLA-A, -B, -C, or -DRB1 on survival after unrelated donor alloSCT. Single mismatches at these loci were associated with significant decrements in survival, and the presence of multiple mismatches was even worse. Low-resolution mismatches appear to have a more severe impact on survival than mismatches detectable only with high-resolution typing techniques, but high-resolution mismatches were also associated with adverse outcomes. ${ }^{78}$

Only $25 \%$ of patients have an HLA-identical sibling. Furthermore, many patients lack HLA-matched unrelated donors. Alternative sources of alloSCT are unrelated umbilical cord blood and HLA-haploidentical relatives. ${ }^{79}$ Extensive graft T-cell depletion has proved effective in preventing GVHD in this context, but can induce a high incidence of infectious complications. ${ }^{79-81}$ Recently, new conditioning strategies of pharmacologic immune suppression, such as the use of high-dose posttransplantation cyclophosphamide, have become more important. Also, in MDS patients, this kind of conditioning is accompanied with success. ${ }^{81}$ In addition, new immunosuppressive strategies in alloSCT with a haploidentical donor lead to better outcomes in MDS patients. ${ }^{80}$

\section{Hypomethylating agents}

New therapeutic alternatives with HMAs have been recently introduced. ${ }^{82}$ HMAs allow the delay of alloSCT by serving as an effective and well-tolerated means to reduce disease burden. HMAs should be considered mainly for older patients with clinically significant comorbidities. ${ }^{4}$ Recently, advantages of pretransplant cytoreductive therapy using HMAs or chemotherapy were demonstrated on the outcome of alloSCT. ${ }^{9}$
Two retrospective studies have demonstrated that pretransplant therapy with 5-azacitidine is associated with less toxicity than induction chemotherapy and may allow for similar outcomes after alloSCT. ${ }^{83,84}$ Treatment with HMAs might also enhance the GVL effect, since preclinical studies have demonstrated that it might lead to an increased expression of KIR and minor histocompatibility antigens, as well as recovery of reduced expression of antigens of tumor cells. ${ }^{4}$ Prospective controlled trials are needed to address optimal initial management of patients with MDS who are candidates for hematopoietic transplantation. Until those data are available, given the acceptable toxicity, potential for cytoreduction, and acceptable transplant-related mortality, HMAs may have an advantage over chemotherapy for MDS patients who are transplant candidates and need further therapy. ${ }^{4}$

\section{Relapse after alloSCT}

Notably, causes of late morbidity and mortality, including GVHD and relapse, remain, and challenge patient care. In the absence of prospective randomized trials, emphasis should be put on patient selection and optimization of preand posttransplant treatment in order to achieve long-term disease control and at the same time maintain an adequate quality of life.

Several therapeutic approaches to control disease, including chemotherapy, withdrawal of immunosuppression, donor lymphocyte infusions, and second alloSCT, can be considered. The optimal treatment strategy for MDS patients relapsing after alloSCT also remains undefined, because prospective trials comparing different approaches are lacking. ${ }^{4}$

The results of a prospective, multicenter German Phase II study investigating the application of single-agent 5-azacitidine followed by donor lymphocyte infusions in patients with AML and MDS relapsing after alloSCT have been promising (remission rate of $32 \%$ ). ${ }^{85}$ Prospective randomized trials are required.

\section{Conclusion}

AlloSCT is the treatment of choice for the majority of MDS patients. With the development of RIC regimens and modified immunosuppression, alloSCT can be offered to older patients, which is very important in view of the age distribution of MDS. Given the growing range of treatment options and the absence of prospective trials, patients need to be stratified according to comorbidities, performance status, and disease risk. Current therapeutic approaches using chemotherapy and HMAs allow the delay of alloSCT by serving as an effective and well-tolerated means to reduce disease burden. 


\section{Disclosure}

The authors report no conflicts of interest in this work.

\section{References}

1. Adès L, Itzykson R, Fenaux P. Myelodysplastic syndromes. Lancet. 2014;383:2239-2252.

2. Greenberg P, Cox C, LeBeau MM, et al. International scoring system for evaluating prognosis in myelodysplastic syndromes. Blood. 1997;89:2079-2088.

3. Garcia-Manero G. Myelodysplastic syndromes: 2014 update on diagnosis, risk-stratification, and management. Am J Hematol. 2014;89:97-108.

4. Platzbecker U, Mufti G. Allogeneic stem cell transplantation in MDS: how? When? Best Pract Res Clin Haematol. 2013;26:421-429.

5. Gooley TA, Chien JW, Pergam SA, et al. Reduced mortality after allogeneic hematopoietic-cell transplantation. N Engl J Med. 2010;363: 2091-2101.

6. Scott BL, Sandmaier BM, Storer B, et al. Myeloablative vs nonmyeloablative allogeneic transplantation for patients with myelodysplastic syndrome or acute myelogenous leukemia with multilineage dysplasia: a retrospective analysis. Leukemia. 2006;20:128-135.

7. Martino R, Valcárcel D, Brunet S, Sureda A, Sierra J. Comparable nonrelapse mortality and survival after HLA-identical sibling blood stem cell transplantation with reduced or conventional-intensity preparative regimens for high-risk myelodysplasia or acute myeloid leukemia in first remission. Bone Marrow Transplant. 2008;41:33-38.

8. Fenaux P, Mufti GJ, Hellstrom-Lindberg E, et al. Efficacy of azacitidine compared with that of conventional care regimens in the treatment of higher-risk myelodysplastic syndromes: a randomised, open-label, phase III study. Lancet Oncol. 2009;10:223-232.

9. Oran B, Kongtim P, Popat U, et al. Cytogenetics, donor type and use of hypomethylating agents in myelodysplastic syndrome with allogeneic stem cell transplantation. Biol Blood Marrow Transplant. Epub June 20, 2014.

10. Sierra J, Pérez WS, Rozman C, et al. Bone marrow transplantation from HLA-identical siblings as treatment for myelodysplasia. Blood. 2002;100:1997-2004.

11. Cutler CS, Lee SJ, Greenberg P, et al. A decision analysis of allogeneic bone marrow transplantation for the myelodysplastic syndromes: delayed transplantation for low-risk myelodysplasia is associated with improved outcome. Blood. 2004;104:579-585.

12. Giagounidis A, Haase D. Morphology, cytogenetics and classification of MDS. Best Pract Res Clin Haematol. 2013;26:337-353.

13. Greenberg PL, Tuechler H, Schanz J, et al. Revised international prognostic scoring system for myelodysplastic syndromes. Blood. 2012;120:2454-2465.

14. Schanz J, Tüchler H, Solé F, et al. New comprehensive cytogenetic scoring system for primary myelodysplastic syndromes (MDS) and oligoblastic acute myeloid leukemia after MDS derived from an international database merge. J Clin Oncol. 2012;30:820-829.

15. Alessandrino EP, Della Porta MG, Bacigalupo A, et al. WHO classification and WPSS predict posttransplantation outcome in patients with myelodysplastic syndrome: a study from the Gruppo Italiano Trapianto di Midollo Osseo (GITMO). Blood. 2008;112:895-902.

16. Breems DA, Van Putten WL, De Greef GE, et al. Monosomal karyotype in acute myeloid leukemia: a better indicator of poor prognosis than a complex karyotype. J Clin Oncol. 2008;26:4791-4797.

17. van Gelder M, de Wreede LC, Schetelig J, et al. Monosomal karyotype predicts poor survival after allogeneic stem cell transplantation in chromosome 7 abnormal myelodysplastic syndrome and secondary acute myeloid leukemia. Leukemia. 2013;27:879-888.

18. Kao JM, McMillan A, Greenberg PL, et al. Impact of cytopenias on clinical outcomes in myelodysplastic syndrome. Am J Hematol. 2008;83:765-770.
19. Malcovati L, Della Porta MG, Strupp C, et al. Impact of the degree of anemia on the outcome of patients with myelodysplastic syndrome and its integration into the WHO classification-based Prognostic Scoring System (WPSS). Haematologica. 2011;96:1433-1440.

20. Cutler C. Allogeneic hematopoietic stem-cell transplantation for myelodysplastic syndrome. Hematol Am Soc Hematol Educ Program. 2010;2010:325-329.

21. Cutler C. Patient selection for transplantation in the myelodysplastic syndromes. Hematol Oncol Clin North Am. 2010;24:469-476.

22. Gyurkocza B, Deeg HJ. Allogeneic hematopoietic cell transplantation for MDS: for whom, when and how? Blood Rev. 2012;26:247-254.

23. Koreth J, Pidala J, Perez WS, et al. Role of reduced-intensity conditioning allogeneic hematopoietic stem-cell transplantation in older patients with de novo myelodysplastic syndromes: an international collaborative decision analysis. J Clin Oncol. 2013;31:2662-2670.

24. Greenberg PL, Attar E, Bennett JM, et al. NCCN Clinical Practice Guidelines in Oncology: myelodysplastic syndromes. J Natl Compr Canc Netw. 2011;9:30-56.

25. Kuendgen A, Strupp C, Aivado M, et al. Myelodysplastic syndromes in patients younger than age 50. J Clin Oncol. 2006;24:5358-5365.

26. Kuendgen A, Gattermann N, Germing U. Improving the prognostic evaluation of patients with lower risk myelodysplastic syndromes. Leukemia. 2009;23:182-184.

27. Oliansky DM, Antin JH, Bennett JM, et al. The role of cytotoxic therapy with hematopoietic stem cell transplantation in the therapy of myelodysplastic syndromes: an evidence-based review. Biol Blood Marrow Transplant. 2009;15:137-172.

28. Kantarjian H, O'Brien S, Ravandi F, et al. Proposal for a new risk model in myelodysplastic syndrome that accounts for events not considered in the original International Prognostic Scoring System. Cancer. 2008;113: $1351-1361$.

29. Mukherjee S, Boccaccio D, Sekeres MA, Copelan E. Allogeneic hematopoietic cell transplantation for myelodysplastic syndromes lingering uncertainties and emerging possibilities. Biol Blood Marrow Transplant. Epub July 29, 2014.

30. Yakoub-Agha I, de La Salmoniere P, Ribaud P, et al. Allogeneic bone marrow transplantation for therapy-related myelodysplastic syndrome and acute myeloid leukemia: a long-term study of 70 patients - report of the French Society of Bone Marrow Transplantation. J Clin Oncol. 2000;18:963-971.

31. Castro-Malaspina H, Harris RE, Gajewski J, et al. Unrelated donor marrow transplantation for myelodysplastic syndromes: outcome analysis in 510 transplants facilitated by the National Marrow Donor Program. Blood. 2002;99:1943-1951.

32. Arnold R, de Witte T, van Biezen A, et al. Unrelated bone marrow transplantation in patients with myelodysplastic syndromes and secondary acute myeloid leukemia: an EBMT survey. European Blood and Marrow Transplantation Group. Bone Marrow Transplant. 1998;21: 1213-1216.

33. Copelan EA, Penza SL, Elder PJ, et al. Analysis of prognostic factors for allogeneic marrow transplantation following busulfan and cyclophosphamide in myelodysplastic syndrome and after leukemic transformation. Bone Marrow Transplant. 2000;25:1219-1222.

34. Martino R, Caballero MD, Pérez-Simón JA, et al. Evidence for a graft-versus-leukemia effect after allogeneic peripheral blood stem cell transplantation with reduced-intensity conditioning in acute myelogenous leukemia and myelodysplastic syndromes. Blood. 2002;100: 2243-2245.

35. Lim Z, Brand R, Martino R, et al. Allogeneic hematopoietic stem-cell transplantation for patients 50 years or older with myelodysplastic syndromes or secondary acute myeloid leukemia. J Clin Oncol. 2010;28: 405-411.

36. Malcovati L, Hellström-Lindberg E, Bowen D, et al. Diagnosis and treatment of primary myelodysplastic syndromes in adults: recommendations from the European LeukemiaNet. Blood. 2013;122: 2943-2964. 
37. Nösslinger T, Tüchler H, Germing U, et al. Prognostic impact of age and gender in 897 untreated patients with primary myelodysplastic syndromes. Ann Oncol. 2010;21:120-125.

38. Zipperer E, Pelz D, Nachtkamp K, et al. The hematopoietic stem cell transplantation comorbidity index is of prognostic relevance for patients with myelodysplastic syndrome. Haematologica. 2009;94:729-732.

39. Della Porta MG, Malcovati L, Strupp C, et al. Risk stratification based on both disease status and extra-hematologic comorbidities in patients with myelodysplastic syndrome. Haematologica. 2011;96:441-449.

40. Sperr WR, Wimazal F, Kundi M, et al. Comorbidity as prognostic variable in MDS: comparative evaluation of the HCT-CI and CCI in a core dataset of 419 patients of the Austrian MDS Study Group. Ann Oncol. 2010;21:114-119.

41. Sorror ML, Maris MB, Storb R, et al. Hematopoietic cell transplantation (HCT)-specific comorbidity index: a new tool for risk assessment before allogeneic HCT. Blood. 2005;106:2912-2619.

42. Zipperer E, Tanha N, Strupp C, et al. The myelodysplastic syndromecomorbidity index provides additional prognostic information on patients stratified according to the revised international prognostic scoring system. Haematologica. 2014;99:e31-e32.

43. Appelbaum FR. The role of hematopoietic cell transplantation as therapy for myelodysplasia. Best Pract Res Clin Haematol. 2011;24: 541-547.

44. Zeidan AM, Linhares Y, Gore SD. Current therapy of myelodysplastic syndromes. Blood Rev. 2013;27:243-259.

45. Malcovati L, Germing U, Kuendgen A, et al. Time-dependent prognostic scoring system for predicting survival and leukemic evolution in myelodysplastic syndromes. J Clin Oncol. 2007;25:3503-3510.

46. Malcovati L, Della Porta MG, Pascutto C, et al. Prognostic factors and life expectancy in myelodysplastic syndromes classified according to WHO criteria: a basis for clinical decision making. J Clin Oncol. 2005;23:7594-7603.

47. Della Porta MG, Malcovati L, Boveri E, et al. Clinical relevance of bone marrow fibrosis and CD34-positive cell clusters in primary myelodysplastic syndromes. J Clin Oncol. 2009;27:754-762.

48. Kröger N, Zabelina T, van Biezen A, et al. Allogeneic stem cell transplantation for myelodysplastic syndromes with bone marrow fibrosis. Haematologica. 2011;96:291-297.

49. Armand P, Kim HT, Cutler CS, et al. Prognostic impact of elevated pretransplantation serum ferritin in patients undergoing myeloablative stem cell transplantation. Blood. 2007;109:4586-4588.

50. Pullarkat V, Blanchard S, Tegtmeier B, et al. Iron overload adversely affects outcome of allogeneic hematopoietic cell transplantation. Bone Marrow Transplant. 2008;42:799-805.

51. Kataoka K, Nannya Y, Hangaishi A, et al. Influence of pretransplantation serum ferritin on nonrelapse mortality after myeloablative and nonmyeloablative allogeneic hematopoietic stem cell transplantation. Biol Blood Marrow Transplant. 2009;15:195-204.

52. Majhail NS, Brunstein CG, McAvoy S, et al. Does the hematopoietic cell transplantation specific comorbidity index predict transplant outcomes? A validation study in a large cohort of umbilical cord blood and matched related donor transplants. Biol Blood Marrow Transplant. 2008;14:985-992.

53. Sivgin S, Baldane S, Kaynar L, et al. Pretransplant serum ferritin level may be a predictive marker for outcomes in patients having undergone allogeneic hematopoietic stem cell transplantation. Neoplasma. 2012;59:183-190.

54. Bejar R, Stevenson K, Abdel-Wahab O, et al. Clinical effect of point mutations in myelodysplastic syndromes. $N$ Engl J Med. 2011;364: 2496-2506.

55. Bejar R, Stevenson KE, Caughey BA, et al. Validation of a prognostic model and the impact of mutations in patients with lower-risk myelodysplastic syndromes. J Clin Oncol. 2012;30:3376-3382.

56. Thol F, Kade S, Schlarmann C, et al. Frequency and prognostic impact of mutations in SRSF2, U2AF1, and ZRSR2 in patients with myelodysplastic syndromes. Blood. 2012;119:3578-3584.
57. Lin CC, Hou HA, Chou WC, et al. IDH mutations are closely associated with mutations of DNMT3A, ASXL1 and SRSF2 in patients with myelodysplastic syndromes and are stable during disease evolution. Am J Hematol. 2014;89:137-144.

58. Saft L, Karimi M, Ghaderi M, et al. p53 Protein expression independently predicts outcome in patients with lower-risk myelodysplastic syndromes with del(5q). Haematologica. 2014;99:1041-1049.

59. Warlick ED, O’Donnell PV, Borowitz M, et al. Myeloablative allogeneic bone marrow transplant using $\mathrm{T}$ cell depleted allografts followed by post-transplant GM-CSF in high-risk myelodysplastic syndromes. Leukoc Res. 2008;32:1439-1447.

60. Warlick ED, Cioc A, Defor T, Dolan M, Weisdorf D. Allogeneic stem cell transplantation for adults with myelodysplastic syndromes: importance of pretransplant disease burden. Biol Blood Marrow Transplant. 2009;15(1):30-38.

61. Alyea EP, Kim HT, Ho V, et al. Impact of conditioning regimen intensity on outcome of allogeneic hematopoietic cell transplantation for advanced acute myelogenous leukemia and myelodysplastic syndrome. Biol Blood Marrow Transplant. 2006;12:1047-1055.

62. Martino R, Iacobelli S, Brand R, et al. Retrospective comparison of reduced-intensity conditioning and conventional high-dose conditioning for allogeneic hematopoietic stem cell transplantation using HLA-identical sibling donors in myelodysplastic syndromes. Blood. 2006;108:836-846.

63. Tauro S, Craddock C, Peggs K, et al. Allogeneic stem-cell transplantation using a reduced-intensity conditioning regimen has the capacity to produce durable remissions and long-term disease-free survival in patients with high-risk acute myeloid leukemia and myelodysplasia. J Clin Oncol. 2005;23:9387-9393.

64. Deeg HJ, Storer B, Slattery JT, et al. Conditioning with targeted busulfan and cyclophosphamide for hemopoietic stem cell transplantation from related and unrelated donors in patients with myelodysplastic syndrome. Blood. 2005;100:1201-1207.

65. Giralt SA, Horowitz M, Weisdorf D, Cutler C. Review of stem-cell transplantation for myelodysplastic syndromes in older patients in the context of the Decision Memo for Allogeneic Hematopoietic Stem Cell Transplantation for Myelodysplastic Syndrome emanating from the Centers for Medicare and Medicaid Services. J Clin Oncol. 2011;29:566-572.

66. Xu F, Deeg HJ. Current status of allogeneic hematopoietic cell transplantation for MDS. Curr Pharm Des. 2012;18:3215-3221.

67. Giralt S. Reduced-intensity conditioning regimens for hematologic malignancies: what have we learned over the last 10 years? Hematology Am Soc Hematol Educ Program. 2005:384-389.

68. Abel GA, KorethJ. Optimal positioning of hematopoietic stem cell transplantation for older patients with myelodysplastic syndromes. Curr Opin Hematol. 2013;20:150-156.

69. Chang C, Storer BE, Scott BL, et al. Hematopoietic cell transplantation in patients with myelodysplastic syndrome or acute myeloid leukemia arising from myelodysplastic syndrome: Similar outcomes in patients with de novo disease and disease following prior therapy or antecedent hematologic disorders. Blood. 2007;110:1379-1387.

70. Slavin S, Nagler A, Naparstek E, et al. Nonmyeloablative stem cell transplantation and cell therapy as an alternative to conventional bone marrow transplantation with lethal cytoreduction for the treatment of malignant and nonmalignant hematologic diseases. Blood. 1998;91:756-763.

71. McSweeney PA, Niederwieser D, Shizuru JA, et al. Hematopoietic cell transplantation in older patients with hematologic malignancies: replacing high-dose cytotoxic therapy with graft-versus-tumor effects. Blood. 2001;97:3390-3400.

72. Maris MB, Niederwieser D, Sandmaier BM, et al. HLA-matched unrelated donor hematopoietic cell transplantation after nonmyeloablative conditioning for patients with hematologic malignancies. Blood. 2003;102:2021-2030.

73. Giralt S. Bone marrow transplant in myelodysplastic syndromes: new technologies, same questions. Curr Hematol Rep. 2005;4:200-207. 
74. Valcárcel D, Martino R, Caballero D, et al. Sustained remissions of high-risk acute myeloid leukemia and myelodysplastic syndrome after reduced-intensity conditioning allogeneic hematopoietic transplantation: chronic graft-versus-host disease is the strongest factor improving survival. J Clin Oncol. 2008;26:577-584.

75. de Witte T, Hermans J, Vossen J, et al. Haematopoietic stem cell transplantation for patients with myelo-dysplastic syndromes and secondary acute myeloid leukaemias: A report on behalf of the Chronic Leukaemia Working Party of the European Group for Blood and Marrow Transplantation (EBMT). Br J Haematol. 2000;110:620-630.

76. Lim ZY, Ho AY, Ingram W, et al. Outcomes of alemtuzumab-based reduced intensity conditioning stem cell transplantation using unrelated donors for myelodysplastic syndromes. Br J Haematol. 2006;135: 201-209.

77. Nakamura R, Rodriguez R, Palmer J, et al. Reduced-intensity conditioning for allogeneic hematopoietic stem cell transplantation with fludarabine and melphalan is associated with durable disease control in myelodysplastic syndrome. Bone Marrow Transplant. 2007;40: 843-850.

78. Flomenberg N, Baxter-Lowe LA, Confer D, et al. Impact of HLA class I and class II high-resolution matching on outcomes of unrelated donor bone marrow transplantation: HLA-C mismatching is associated with a strong adverse effect on transplantation outcome. Blood. 2004;104: 1923-1930.

79. Aversa F, Terenzi A, Tabilio A, et al. Full haplotype-mismatched hematopoietic stem-cell transplantation: a phase II study in patients with acute leukemia at high risk of relapse. J Clin Oncol. 2005;23: 3447-3454.
80. Peccatori J, Forcina A, Clerici D, et al. Sirolimus-based graft-versushost disease prophylaxis promotes the in vivo expansion of regulatory $\mathrm{T}$ cells and permits peripheral blood stem cell transplantation from haploidentical donors. Leukemia. Epub June 4, 2014.

81. Bashey A, Zhang X, Sizemore CA, et al. T-cell-replete HLA-haploidentical hematopoietic transplantation for hematologic malignancies using posttransplantation cyclophosphamide results in outcomes equivalent to those of contemporaneous HLA-matched related and unrelated donor transplantation. J Clin Oncol. 2013;31:1310-1316.

82. Fenaux P, Adès L. How we treat lower risk myelodysplastic syndromes. Blood. 2013;121:4280-4286.

83. Damaj G, Duhamel A, Robin M, et al. Impact of azacitidine before allogeneic stem-cell transplantation for myelodysplastic syndromes: a study by the Société Française de Greffe de Moelle et de ThérapieCellulaire and the Groupe-Francophone des Myélodysplasies. J Clin Oncol. 2012;30:4533-4540.

84. Gerds AT, Gooley TA, Estey EH, Appelbaum FR, Deeg HJ, Scott BL. Pretransplantation therapy with azacitidine vs induction chemotherapy and posttransplantation outcome in patients with MDS. Biol Blood Marrow Transplant. 2012;18:1211-1218.

85. Schroeder T, Czibere A, Kröger N, et al. Phase II study of azacitidine $\left(\right.$ Vidaza $\left.^{\circledR}, \mathrm{Aza}\right)$ and donor lymphocyte infusions (DLI) As first salvage therapy in patients with acute myeloid leukemia (AML) or myelodysplastic syndromes (MDS) relapsing after allogeneic hematopoietic stem cell transplantation (allo-SCT): final results from the AZARELA-trial (NCT-00795548). Blood. 2011;118:300.
Stem Cells and Cloning: Advances and Applications

\section{Publish your work in this journal}

Stem Cells and Cloning: Advances and Applications is an international, peer-reviewed, open access journal. Areas of interest in stem cell research include: Embryonic stem cells; Adult stem cells; Blastocysts; Cordblood stem cells; Stem cell transformation and culture; Therapeutic cloning; Umbilical cord blood and bone marrow cells; Laboratory,

\section{Dovepress}

animal and human therapeutic studies; Philosophical and ethical issues related to stem cell research. This journal is indexed on CAS. The manuscript management system is completely online and includes a quick and fair peer-review system. Visit http://www.dovepress.com/ testimonials.php to read real quotes from published authors. 